\title{
Gesundheit im Wandel
}

\section{Adrian Ritter}

Freier Journalist

\author{
Die Evolutionäre Medizin sucht mit dem Blick in die Vergangenheit nach den \\ Ursachen und möglichen Ansätzen zur Behandlung heutiger Krankheiten. Im \\ August fand in Zürich der 1. Europäische Kongress der Evolutionsmediziner statt.
}

Was haben Rückenschmerzen mit dem aufrechten Gang des Menschen zu tun? Wie hat sich der Tuberkulose-Erreger im Laufe der Jahrhunderte verändert? Die Evolutionäre Medizin erforscht die Geschichte von Krankheit und Gesundheit und versucht daraus Schlüsse zu ziehen für die Prävention und Behandlung heutiger Krankheiten.

So vielfältig wie die Fragestellungen sind, ist auch die akademische Verortung: Das interdisziplinäre Feld vereint unter anderem Forschende aus Evolutionsbiologie, Archäologie, Public Health, Immunologie und Paläopathologie. Epidemiologische Studien gehören ebenso zu den Methoden der Evolutionären Medizin wie etwa die Analyse der DNA früherer Menschen und die vergleichende Anatomie.

Die Evolutionäre Medizin ist ein junges Forschungsfeld, entstanden in den USA in den 1990er Jahren. Anfang August fand in Zürich der erste Europäische Kongress statt, organisiert vom Institut für Evolutionäre Medizin der Universität Zürich. Dabei präsentierte

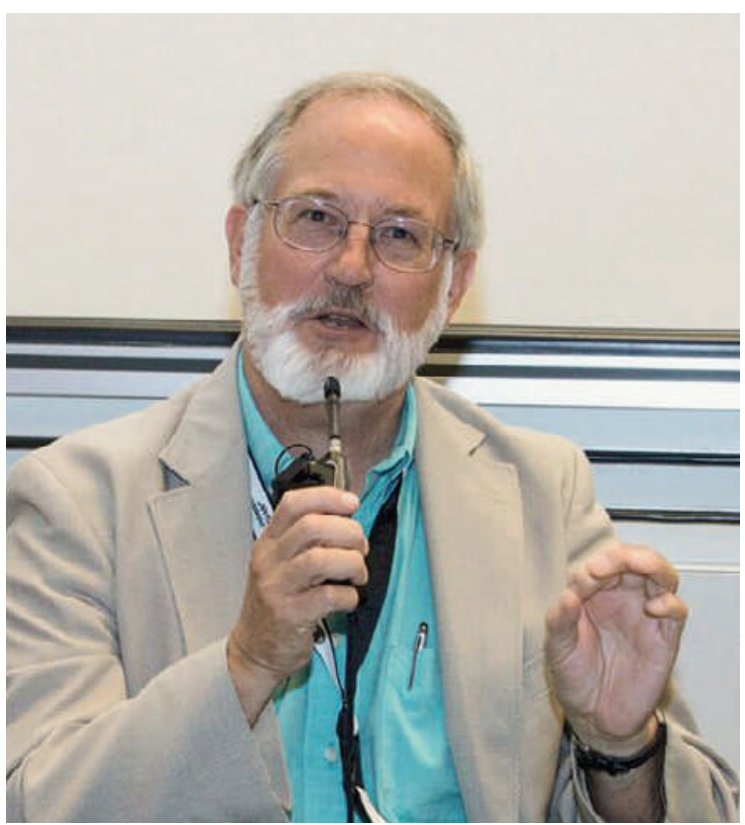

"Medizin ohne Evolution ist wie Ingenieurwesen ohne Physik», so Randolph Nesse, US-Psychiater, Evolutionsbiologe und einer der Begründer der Evolutionären Medizin. sich eine selbstbewusste akademische Gemeinschaft: «Medizin ohne Evolution ist wie Ingenieurwesen ohne Physik», sagte Randolph Nesse, US-Psychiater, Evolutionsbiologe und einer der Begründer der Evolutionären Medizin.

\section{Neue Ideen sind gefragt}

Interdisziplinär und mit breitem historischen Blick will die Evolutionäre Medizin das Sichtfeld der hoch-

In multifaktoriellen Modellen soll das Zusammenspiel von Genetik, Krankheitserregern und anderen Umwelteinflüssen aufgedeckt werden.

spezialisierten heutigen Medizin weiten. Das Interesse gilt dabei sowohl der Entwicklung des menschlichen Körpers, seiner Krankheiten und seines Immunsystems wie der Entwicklung der Krankheitserreger und Umweltbedingungen im Laufe der Menschheitsgeschichte.

Der Anspruch ist hoch: In multifaktoriellen Modellen soll das Zusammenspiel von Genetik, Krankheitserregern und anderen Umwelteinflüssen aufgedeckt werden. Dabei spielen Grundbegriffe aus der Evolutionsbiologie wie Selektion, Anpassung und Variabilität eine zentrale Rolle. Soziale, klimatische und andere Umweltfaktoren führen zu genetischen und morphologischen Anpassungen im Menschen, so eine Grundidee. Krankheiten entstehen unter anderem aufgrund einer fehlerhaften Anpassung an die Umwelt. Denkbar sind auch Faktoren, die früher einen Selektionsvorteil brachten, heute aber krank machen.

\section{Genetik, Erreger und Umwelt}

Auf der Suche nach den weiter zurückliegenden Ursachen von Erkrankungen stellt die Evolutionäre Medizin auch bisherige Ansichten in Frage - etwa diejenige, dass Gefässverkalkung vor allem eine moderne Zivilisationskrankheit sei. Frank Rühli, Medizinprofessor und Direktor des Instituts für Evolutionäre Medizin an 


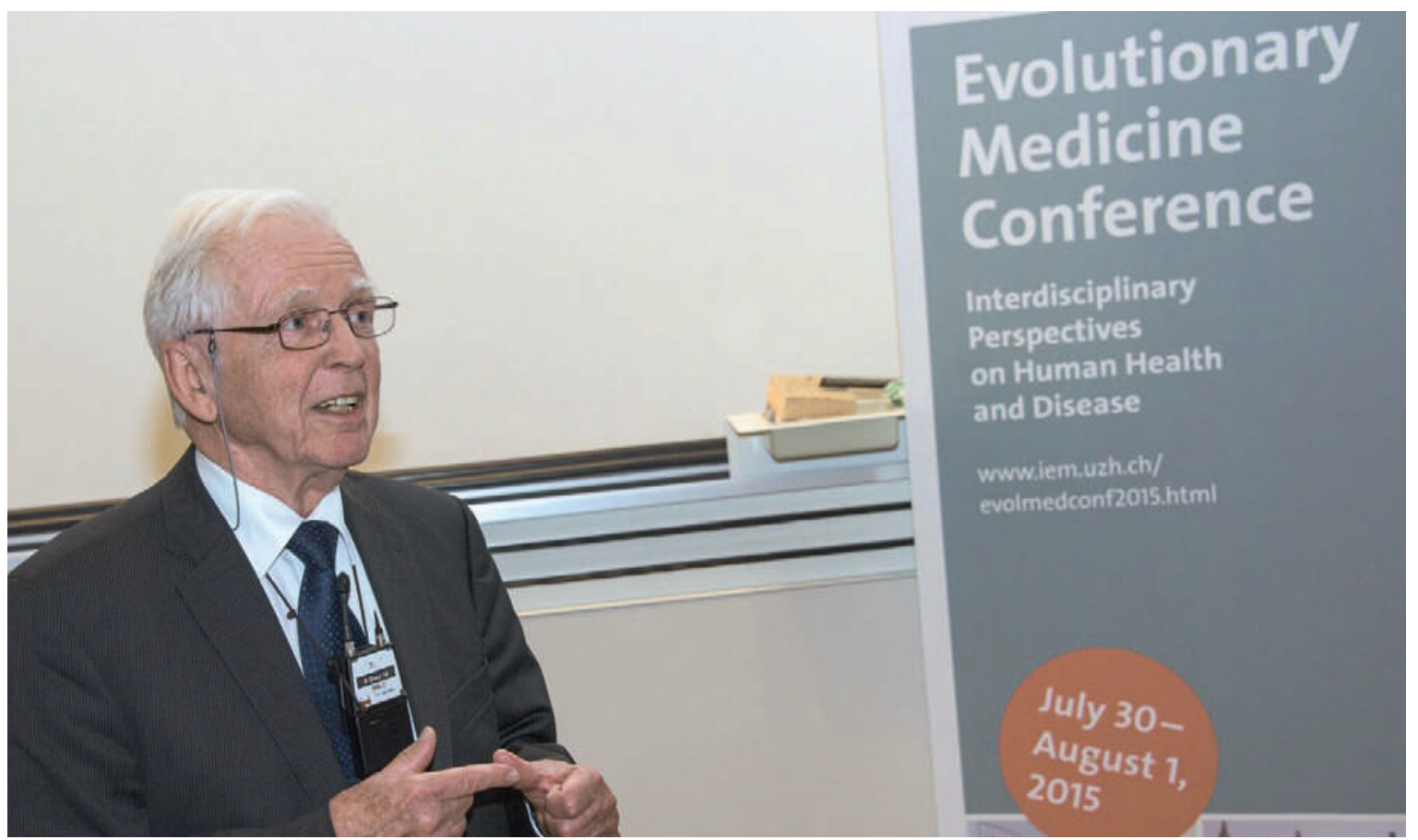

Nobelpreisträger Harald zur Hausen stellte seine aktuelle Forschung zu Darm- und Brustkrebs vor.

der Universität Zürich, findet bei Untersuchungen altägyptischer Mumien immer wieder solche Gefässverkalkungen. Gleichzeitig ist aus historischen Quellen aber bekannt, dass sich die damalige Ernährung stark von der heutigen unterscheidet. Was also ist die Ursache von Gefässverkalkung?

Der US-Evolutionsbiologe Paul Ewald stellte in seinem Referat am Kongress nicht in Frage, dass (Passiv-)Rauchen, Alkohol, eine fettreiche Ernährung sowie genetische Faktoren zu Arterienverkalkung und damit Herzinfarkten beitragen. Seine Forschung zeigt aber, dass alles noch komplexer sein könnte, indem vermutlich zusätzlich ein Bakterium mitspielt.

\section{Institut für Evolutionäre Medizin}

Die Universität Zürich unterhält mit dem 2014 gegründeten Institut für Evolutionäre Medizin (IEM) an der Medizinischen Fakultät eines der wenigen Institute weltweit, die sich ausschliesslich dem Thema Evolutionäre Medizin widmen. Das IEM hat dabei drei thematische Schwerpunkte:

1. Molekulare Evolutionäre Medizin (mittels Erbsubstanz aus Mumien und Skeletten die Entwicklung von Krankheitserregern wie Viren und Bakterien und funktionelle Allelhäufigkeiten studieren);

2. Radiodiagnostik (mittels Bildgebung beispielsweise Krankheiten in Mumien und Skeletten diagnostizieren);

3. Mikroevolution (Veränderungen der menschlichen Anatomie wie den Bau der Wirbelsäule und Krankheitsmuster studieren).

Neue Publikation zum Thema: Stearns SC, Medzhitov R. Evolutionary Medicine. Sunderland: Sinauer Associates Inc.; 2015.
Der Bedeutung von infektiösen Erregern ist auch Harald zur Hausen auf der Spur. 2008 erhielt er den Medizin-Nobelpreis für die Bestätigung der Hypo-

Gerade bei Zivilisationskrankheiten lohnt sich die langfristige Perspektive auf die Krankheitsursachen.

these, dass humane Papillomviren bei Gebärmutterhalskrebs eine wichtige Rolle spielen. Am Kongress für Evolutionäre Medizin präsentierte er seine aktuelle Forschung zu Darm- und Brustkrebs. Er vermutet, dass ein infektiöser Erreger in der Kuhmilch diese Krebsarten mitverursacht.

\section{Zivilisationskrankheiten neu denken}

Die Vertreterinnen und Vertreter der Evolutionären Medizin sind überzeugt, dass sich gerade bei Zivilisationskrankheiten die langfristige Perspektive auf die Krankheitsursachen lohnt. So hänge die weltweite Zunahme an übergewichtigen Menschen auch damit zusammen, dass es früher ein Selektionsvorteil war, grosse Mengen an Energie speichern zu können für härtere Zeiten. Inzwischen hat sich diese Fähigkeit wohl in einen Nachteil verwandelt.

Oder das Beispiel Rückenschmerzen: Der aufrechte Gang verhalf den Sammlern und Jägern zu einem breiteren Nahrungsangebot, verursacht aber wegen der stärkeren Belastung der Wirbelsäule auch Rücken- 


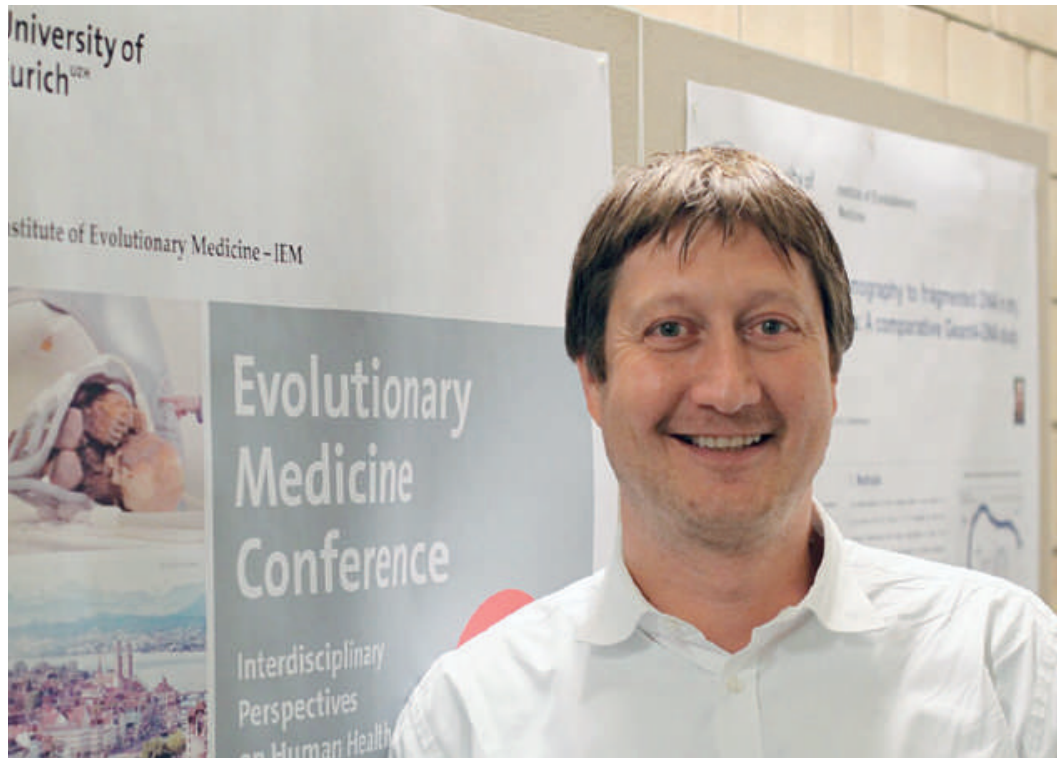

Schon unsere Vorfahren, die Australopithecinen, hatten Rückenschmerzen, vermutet das Team um Frank Rühli aufgrund der Untersuchung von Fossilien.

schmerzen. Schon unsere Vorfahren, die Australopithecinen, könnten darunter gelitten haben, wie Forschende aus dem Team von Frank Rühli aufgrund der Untersuchung von Fossilien vermuten.

\section{Zunehmende Variabilität}

«In der Evolution des Menschen bestanden immer wieder Zielkonflikte. Der menschliche Körper ist ein Kompromissprodukt», sagt Rühli im Gespräch mit der Schweizerischen Ärztezeitung. Zumindest in Ländern mit einer guten Gesundheitsversorgung finde die natürliche Selektion allerdings nur noch sehr eingeschränkt statt.

Die Rede ist von einer «relaxed natural selection»: Auch kranke Menschen überleben und die generelle präpubertale Mortalität sinkt. "Dank In-vitroFertilisation können sich heute glücklicherweise sogar Personen fortpflanzen, die dazu natürlicherweise nicht in der Lage wären", so Rühli. Weil der Selektionsdruck sinke, nehme die genetische und morphologische Variabilität der Menschheit wohl zu: «Der Körper kann sich eher Varianten erlauben, wenn er ohnehin überlebt.»

Gemäss Rühli wird sich dieser Trend tendenziell fortsetzen. Kommende Generationen von Medizinstudierenden werden beim Sezieren von Leichen vermutlich mit mehr Variation konfrontiert werden. Von Anomalien will er dabei nicht reden, im Gegenteil: «Die Evolutionäre Medizin schärft den Blick für die Variabilität des Menschen. Die breitere Norm sollte Toleranz fördern.» Umso wichtiger sei für die Zukunft eine perso- nalisierte Medizin, die auf diese Unterschiede Rücksicht nimmt.

\section{Gelangweiltes Immunsystem}

Die Evolutionäre Medizin steht mit ihrer Forschung erst am Anfang. Für Pionier Randolph Nesse ist klar: «Die Evolutionäre Medizin kann uns heute nicht viel dazu sagen, wie ein Patient behandelt werden soll. Sie kann aber aufzeigen, wie wir über ein bestimmtes Problem nachdenken und welche Forschung wir dazu machen sollten.»

Für Frank Rühli ist allerdings klar, dass Ärztinnen und Ärzte unbewusst durchaus Gedanken der Evolutionären Medizin auch in der Praxis aufnehmen. So sollte etwa das evolutionäre Verständnis von AntibiotikaResistenzbildung helfen, einen optimalen Einsatz von Antibiotika zu ermöglichen und weiteren Resistenzbildungen entgegenzuwirken. Paläogenetische Untersuchungen hätten zudem bereits zum verbesserten Verständnis der Evolution und damit der Virulenz und Umweltsensitivität von Pathogenen wie Tuberkulose beigetragen.

Auch beim Umgang mit dem Thema Hygiene kann der evolutionäre Blick sinnvoll sein. Hygiene ist selbstverständlich unerlässlich, wenn es etwa darum geht, die Ausbreitung von Krankenhauskeimen zu verhindern. Gleichzeitig sei es aber beispielsweise auch wichtig, Kinder in der Natur spielen zu lassen, so Frank Rühli. Gemäss der Hygiene-Hypothese könnte die Zunahme von Autoimmunkrankheiten und Allergien nämlich damit zusammenhängen, dass die Menschen früher

In unserer reinlichen Umgebung wird es dem Immunsystem sozusagen langweilig, es richtet sich gegen den eigenen Körper.

viel stärker Mikroben ausgesetzt waren. In unserer heutigen reinlichen Umgebung werde es dem Immunsystem sozusagen langweilig und es richte sich vermehrt gegen den eigenen Körper.

Die Denkweise der Evolutionären Medizin vermehrt ins Medizinstudium einzubringen ist den Vertreterinnen und Vertretern der Evolutionären Medizin ein grosses Anliegen, wie am Kongress deutlich wurde. Sie soll die Studierenden für die Variabilität sensibilisieren und das kritische Denken fördern. Ein Denken, das Vergangenheit und Gegenwart zu verstehen hilft und in der Zukunft vielleicht auch zu neuen Behandlungsansätzen führt.

Bildnachweise

Bild Rühli: Adrian Ritter; alle anderen Bilder: Corina Steiner 